\title{
Household approach to health related behavioral risks and life patterns among Dubai population
}

\begin{abstract}
Background: Evidence-based practice revealed that, health-related behavioral risks and lifestyle factors significantly contributed to certain NonCommunicable Diseases (NCDs) such as diabetes, mellitus cardiovascular illnesses, and some types of cancer.

Objectives: To study the prevalence and profile of health-related behavioral risks factors includes tobacco use, physical activity, obesity \& overweight as well as healthy food consumption among a representative sample of Dubai population.

Methodology: Dubai Household Health Survey was carried out in 2014 as a crosssectional, multistage, stratified, cluster survey. Houses were visited to obtain detailed information on the different health-related issues. About 4800 families were randomly selected by stratified cluster sampling techniques. With a response rate of $80 \%$, the final sample equals to 13,299 individuals. The design and methodology of the survey were adapted from those used in the World Bank's Living Standards Measurement Surveys (LSMS) and the World Health Organization's World Health Surveys (WHS). Data collection was made by 75 well-trained researchers and 25 well-trained nurses. The duration of data collection lasted to 40 working days under strict technical and administrative procedure. Data was collected electronically using well calipered tablets. Data entry, coding, cleaning, weighing and analysis have been carried out using the SPSS program.
\end{abstract}

Findings: The present study revealed that among the 13,299 respondents, $72.4 \%$ were males and $7.9 \%$ were Emirati. The study showed that proportion of current smoker was $16.1 \%$ (it was $20.1 \%$ for males and $2.9 \%$ for females).

The survey findings underlined a suboptimal intake of fruits and vegetables in the Duba population. Overall, $90.4 \%$ of the sample reported consuming a fruit $1-3$ times a day. At the same context, $90.6 \%$ of the survey population took a vegetable 1 to 3 times a day. In concern with the physical activities, an alarming high prevalence of physical inactivity among the sampled population of Dubai was observed (85.1\%), with $95.4 \%$ of them didn't perform vigorous intensity activities, other than those done as part of work (considered inactive). In addition, only $18.2 \%$ of the survey population did moderate-intensity activities for at least 10 minutes at a time, as part of work (1-3 days per week), and hence considered inactive.

Conclusions: Multiple behavioral risk factors were significantly highlighted among Dubai Population, pointing out physical in-activity at the population level, high consumption of non-healthy food and high levels of tobacco use. This indicates true epidemiological transitions which necessitate aggressive multidimensional interventions and policy actions.

Keywords: behavioral, risks, Dubai, population, household health, survey
Volume 6 Issue 4 - 2019

\author{
Hamid Yahya Hussain,' Heba M Mamdouh,' \\ Fatheya Fardallah Al Awadi, ${ }^{3}$ Mohamed \\ Mahmoud Aly Hassanien, ${ }^{3}$ Eldaw abdalla \\ Mohamed Ali Suliman, ${ }^{4}$ \\ 'Research Studies \&data analysis, DHA \\ ${ }^{2}$ Family Health Dept., HIPH, Alexandria University. \\ ${ }^{3}$ Endocrinology Dept. , Dubai Hospital, DHA \\ ${ }^{4}$ Strategies \&governance Dept. DHA
}

Correspondence: Dr Hamid Yahya Hussain, Department of Health Affairs, Dubai Health Authority, UAE, Email hussainh569@gmail.com

Received: July II, 2019 | Published: September 30, 2019

\section{Introduction}

Evidence-based practice revealed that health-related behavioral risks and unhealthy lifestyle factors could significantly contribute to certain non-communicable diseases (NCDs) such as diabetes mellitus, ${ }^{1,2}$ cardiovascular illnesses, ${ }^{2,3}$ chronic respiratory diseases, and some types of cancer. ${ }^{1-6} \mathrm{NCDs}$ are now recognized as the leading causes of death in most regions of the world. Annually, sixteen million
NCD deaths occur before the age of $70 ; 82 \%$ of these "premature" deaths occurred in low and middle-income countries., ${ }^{3,9}$ These diseases share four modifiable key risk factors; tobacco use, use of alcohol, physical inactivity, and unhealthy eating habits. ${ }^{2,6,9}$ In the emirate of Dubai, NCDs are already responsible for more than half of all deaths. ${ }^{10}$ Unless urgent action is taken, the growing NCD epidemic will add tremendous pressure to the overstretched health systems and pose a major challenge to the future heath agenda. 
Currently, healthcare systems all over the world are encountering major challenges of reducing the burden of diseases and early death from the NCDs. Monitoring the population often makes a good base for implementing evidence-based preventive and promotional programs. ${ }^{3,11}$ Monitoring trends in life style factors and scaling up proven, cost-effective interventions are important strategies for addressing NCDs. Accordingly; this paper will shed light on some behavioral risk factors that shared by a representative sample of Dubai population.

\section{Methodology}

The survey was carried out as a cross-sectional, multistage, stratified cluster survey. Houses were visited to obtain detailed information on the different health-related issues. About 4800 families were randomly selected by stratified cluster sampling techniques. With a response rate of $80 \%$, the final sample equals to 13,299 individuals. The design and methodology of the survey were adapted from those used in the World Bank's Living Standards Measurement Surveys and the World Health Organization's World Health Surveys.

Data were collected by field workers and nurses who were recruited and trained specially for the purposes of the survey. Training was on data collection techniques and logistics, and Specific issues regarding individual components of the questionnaires that include; NCD, elderly health, behavioral risk Factors and healthcare expenditure. The data collection phase was carried out for about 2 months during the year 2014 under strict technical and administrative and auditing procedures. Data entry was implemented in the field using tables. Data were aggregated centrally and SPSS data sheet was created by Dubai Statistics Center. Data coding, entry cleaning, weighing and analysis has been carried out using SPSS software.

\section{Results}

\section{Socioeconomic background}

Among the respondents, $72.4 \%$ were males and $7.9 \%$ were Emirati. According to survey data, a quarter of the sample $(25 \%)$ was under 20 years; with most of the surveyed population was less than 45 years $(83.1 \%)$. As for the education status, Illiterates constituted only $3 \%$ of those in the age group 18-59 years, compared to $18 \%$ among those in the age group $60+$ years. The majority of the respondents $(69.6 \%)$ were currently married and $99.4 \%$ had health insurance coverage (data was not shown in the tables).

\section{Lifestyles and health behaviors}

Overall proportion of current smokers within the surveyed population (daily and not daily) was $16.1 \%$ (20.1\% for males, and $2.9 \%$ for females). Tobacco use has been reported to be highest in young men between the ages of 25-44 (23\%), as shown in Table 1. The proportion of non-UAE who reported to use tobacco greatly exceeded that proportion of UAE-nationals ( $17 \% \& 7.2 \%$, respectively). Most current smokers were actually daily smokers $(68.3 \%)$, while the proportion of non-daily smokers in men was only $7.8 \%$. The duration of smoking among the survey population was 20 years and more in $18.9 \%$ of cases. In concern with physical activities, an alarming high prevalence of physical inactivity among the sampled population of Dubai was observed (85.1\%), with $15.2 \%$ of males and 13.75 of females among the sample reported to be physically activate (Table 2). The surveyed non-UAE nationals were more active than UAEnationals $(15 \%$ \& $13 \%$, respectively). Asking about the time spend doing different types of physical activity in a typical week as part of work (by work we mean paid and unpaid work, as well as house work) was an important component of the survey. Overall, $18.2 \%$ of the survey population do moderate-intensity activities for at least 10 minutes at a time for 1 to 3 days per week as part of work and hence considered inactive (Figure 1). When they were asked if they do any vigorous intensity activities, other than those done as part of work (like running or playing football), for at least 10 minutes continuously, almost all the sample (95.4\%) did not do that and hence considered inactive. Moreover, $81.9 \%$ of the surveyed population did not do any moderateintensity activities, other than those done as part of work, that cause a small increase in breathing or heart rate (such as brisk walking) for at least 10 minutes at a time and hence considered inactive (Data was not shown in the tables).

The survey findings underlined a suboptimal intake of fruits and vegetables among the Dubai population. A fruit or vegetables serving was identified as a medium sized fruit/ vegetables, or a handful of smaller fruits, fresh or dried. Overall, $90.4 \%$ of the sample reported consuming a fruit 1-3 times a day (Figure 2). At the same context, $90.6 \%$ of the survey population took a vegetable 1 to 3 times a day (Figure 3). Regarding the other eating habits, 26\% of the sampled population answered that they eat animal products 3 times per day, $83.2 \%$ of them eat 3 meals daily, $69.4 \%$ drank $8+$ water glasses per day and the majority (92\%) did take their breakfast (Data was not shown).

Table I Prevalence of current tobacco use (\%) among adults (18+ years) by age and gender

\begin{tabular}{llll}
\hline Male & Female & Total & Age Group \\
\hline 15.3 & 1.7 & 11.8 & $18-24$ \\
22.8 & 3.7 & 18.5 & $25-44$ \\
17.4 & 1.6 & 13.8 & $45-64$ \\
13.2 & 5.7 & 10.6 & $65+$ \\
20.1 & 2.9 & 16.1 & Total
\end{tabular}

Table 2 Prevalence of physical activity (\%) among adults (18+ years) by age and gender

\begin{tabular}{llll}
\hline Male & Female & Total & Age Group \\
\hline 13.5 & 9.4 & 12.5 & $18-24$ \\
13.9 & 15.1 & 14.2 & $25-44$ \\
18.4 & 13.5 & 17.3 & $45-64$ \\
13.9 & 12.5 & 13.4 & $65+$ \\
15.2 & 13.8 & 14.9 & Total
\end{tabular}




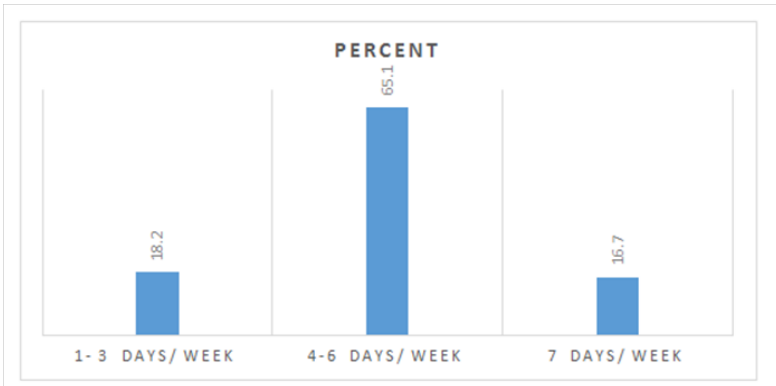

Figure I Moderate intensity activity as part of work per week among adults (18+ years).

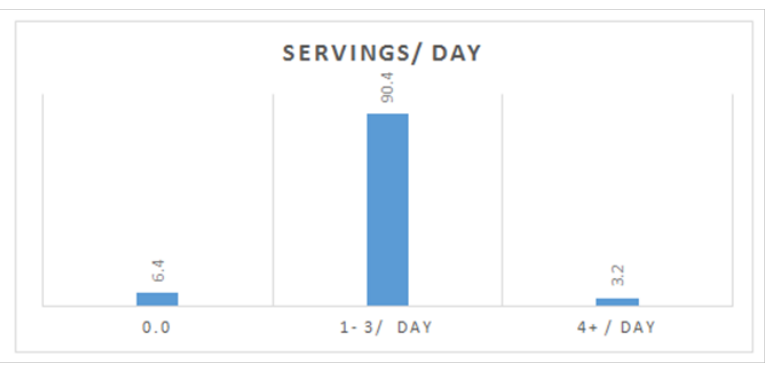

Figure 2 Fruits Consumption per day among adults (18+ years).

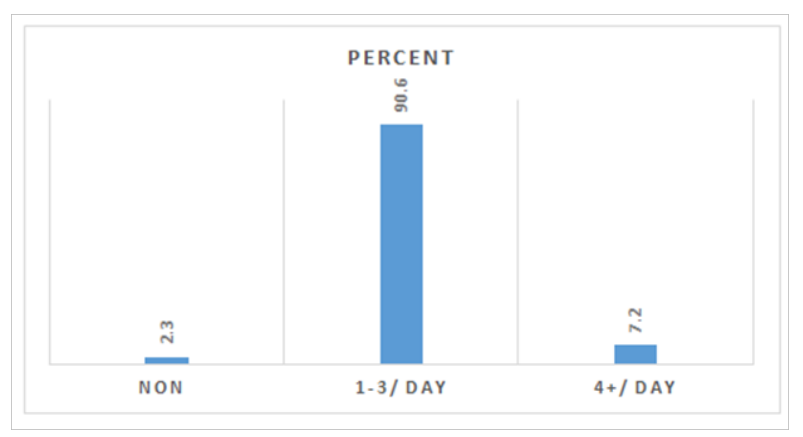

Figure 3 Vegetables consumption per day among adults (18+ years).

\section{Discussions}

The current data generated from the 2014 Dubai household survey, have reported a high prevalence of behavioral risk factors that pose a significant threat to the Dubai population. Some of the findings concerning tobacco use, inadequate fruit and vegetables consumption and physical inactivity have been found to be higher than the figures reported by others studies conducted among other Arab countries. ${ }^{12-16}$ Some gender and socioeconomic differentials within several factors have also been identified. This is reflecting a serious potential of NCD morbidities and mortalities within the coming decades. Among the sampled population, overall proportion of current smokers within the surveyed population (daily and not daily) was $16.1 \%$; with male smokers over numbered female smokers. However, it can be assumed that many young adults and youth in particular might be not able to disclose their real smoking status at a household survey setting. It is worth mentioning that the first study in the region to biochemically verify tobacco use self-report data revealed a much higher alarming prevalence of Tobacco use in the UAE. Self-reported tobacco use was
$36 \%$ among men and 3\% among women in the sample. ${ }^{17}$ Moreover, biochemical verification of smoking status revealed that $42 \%$ men and $9 \%$ of women were positive for nicotinine indicating possible recent tobacco use indicating that misclassification of smoking status was more common than expected. Efforts are needed to really monitor tobacco use and adopt the most prevention policies in place. In accordance with the current reported tobacco use, among a surveyed sample in Saudi Arabia, the prevalence of cigarette smoking was $12.7 \%$; males smoke more frequently than females (19.0\% vs. $4.0 \%)$. The Saudi Arabian study also found that male smokers exercised significantly less than nonsmokers. ${ }^{18}$

In the present study, average consumptions of fruits and vegetables revealed inadequate intake and as per the standard recommendations. ${ }^{19}$ The guidelines stated that for people ages 19 to 30 , the recommended daily amount of vegetables for men is at least 3 cups a day and for women, at least $2^{1 / 2}$ cups. It has been suggested that diets high in saturated fat, and low in fruit and vegetables are likely to be associated with the increased risk of heart disease, stroke, obesity and some cancers. ${ }^{1,5,20-22}$ Though dietary habits are often rooted in local and regional traditions, strategies aimed at improving dietary habits, therefore, can play a key part in reducing early deaths from these diseases. ${ }^{11}$ National strategies, therefore, need to be culturally appropriate and able to challenge cultural influences and to change over time. Promoting a healthy diet requires a multi-sectoral approach. There is a need for close co-operation among health, agriculture and food industry sectors. Strategies for the adoption of a healthy diet include marketing, advertising, sponsorship and promoting food products consistent with a healthy diet, fiscal policies that can influence price through taxation, subsidies or direct pricing encouraging healthy eating, development of dietary guidelines, education, communication for public awareness, etc. ${ }^{23}$ 'Five-a-day' campaign is an important initiative adopted in parts of Europe, the principal objective of the initiative is to increase consumption levels of a variety of different fruit and vegetables to at least five portions (400g) per day. ${ }^{24}$ Low physical activity is considered an important predictor of many chronic diseases, most notably heart disease, stroke, obesity, type 2 diabetes and some types of cancers. ${ }^{9}{ }^{913}$ Beyond its role in the development of obesity, physical inactivity and associated poor cardio-respiratory fitness pose direct health risks., ${ }^{2,13}$ The global estimate for the prevalence of physical inactivity among adults is $17 \%$ compared with the observed rate of $85 \%$ among the current sample. ${ }^{2}$ However, data on the physical activity from the current sample showed that the current prevalence rate is quite similar to that have been reported within other GCC countries according to the systematic review study carried out 2012. ${ }^{13}$ The systemic review stated that, In most countries, inactivity exceeded $40 \%$; indicating that throughout the region, levels of physical inactivity are very high; Kuwait (62.6\%), Saudi Arabia (67.6\%), and Qatar (45.9\%). The review also highlighted that, among children and adolescents, inactivity is alarmingly high.

\section{Conclusions}

Multiple behavioral risk factors were highlighted among this sample of Dubai Population, pointing out worrying results for physical in-activity at the population level, high consumption of nonhealthy food and high levels of tobacco use. This evidence indicating true epidemiological transition, which necessitate aggressive multidimensional intervention and multi sector collaboration. 
Lifestyle and behavioral-based programs at the national level have to be at the top of national health agendas. National framework needs to be established, through involvement of relevant stakeholders under the lead of different health authorities, to put an action plan aiming at lowering those behavioral risk factors at the country level.

\section{Ethical issues}

Ethical standards have been adhered to throughout the work conduction.

\section{Acknowledgments}

None.

\section{Conflicts of interest}

All Authors declared that there were no conflicts of interest to this point of progress.

\section{References}

1. Parkin DM, Boyd L. Cancers attributable to overweight and obesity in the UK in 2010. Br J Cancer. 2011;105(Supply 2):34-37.

2. World Health Organization. Global Health Risks mortality and burden of disease attributable to selected major risks. Geneva: World Health Organization. 2009.

3. World Health Report. Reducing risks, promoting a healthy life. Geneva: World Health Organization. 2002

4. Lauby Secretan B, Scoccianti C, Loomis D, et al. Body fatness and cancer-viewpoint of the IARC Working Group. $N$ Engle $J$ Med. 2016;375(8):794-798.

5. Parkin DM. Cancers are attributable to inadequate physical exercise in the UK in 2010. Br J Cancer. 2011;105(Supply 2):38-41.

6. Parkin DM. Tobacco attributable cancer burden in the UK in 2010. Br J Cancer. 2011;105(Supply 2):6-13.

7. WHO. No communicable Diseases Fact Sheet. 2018.

8. Peto R, Lopez AD, Boreham J, et al. Mortality from Smoking in Developed Countries, 1950-2000: Indirect Estimates from National Statistics. Lancet. 1992;339(8804):1268-1278.

9. Lee IM, Shiroma EJ, Lobelo F, et al. Effect of physical inactivity on major non-communicable diseases worldwide: an analysis of burden of disease and life expectancy. Lancet. 2012;380(9838):219-229.
10. Dubai Annual Health Statistics Report. 2017.

11. WHO. Global Action Plan for the Prevention and Control of NCDs 2013 2020. 2013

12. Aleissa EL. The Frequency of Health-Related Behaviors Among Saudi Adolescents Visiting Primary Health Care Centers In Riyadh City. $J$ Family Community Med. 2001;8(1):19-26.

13. Sharara E, Akik C, Ghattas H, et al. Physical inactivity, gender and culture in Arab countries: a systematic assessment of the literature. BMC Public Health. 2018;18(1):639.

14. Chacar HR, Salameh P. Public schools adolescents' obesity and growth curves in Lebanon. J Med Liban. 2011;59(2):80-88.

15. Mabry R, Winkler E, Reeves M, et al. Correlates of Omani adults' physical inactivity and sitting time. Public Health Nutr. 2013;16(1):65-72.

16. Mahfouz AA, Shatoor AS, Khan MY, et al. Nutrition, physical activity, and gender risks for adolescent obesity in Southwestern Saudi Arabia. Saudi J Gastroenterol. 2011;17(5):318-322.

17. Al Houqani M, Leinberger Jabari A, Al Naeemi A, et al. Patterns of tobacco use in the United Arab Emirates Healthy Future (UAEHFS) pilot study. PLoS One. 2018;13(5):0198119.

18. Maziar Moradi Lakeh, Charbel El Bcheraoui, Marwa Tuffaha, et al. Tobacco consumption in the Kingdom of Saudi Arabia, 2013: findings from a national survey. BMC Public Health. 2015;15:611.

19. Lehman S. How Many Vegetables Should You Eat Everyday? 2019.

20. Al Nakeeb Y, Lyons M, Collins P, et al. Obesity, physical activity and sedentary behavior amongst British and Saudi youth: a cross-cultural study. Int J Environ Res Public Health. 2012;9(4):1490-1506.

21. Aounallah Skhiri H, Ben Romdhane H, Maire B, et al. Health and behaviors of Tunisian school youth in an era of rapid epidemiological transition. East Mediterr Health J. 2009;15(5):1201-1214.

22. Sibai AM, Costanian C, Tohme R, et al. Physical activity in adults with and without diabetes: from the 'high-risk' approach to the 'populationbased' approach of prevention. BMC Public Health. 2013;13:1002.

23. World Health Organization. Diet, nutrition and the prevention of chronic diseases: Report of a joint WHO/FAO expert consultation. 2003.

24. Health survey for England: Fruits and Vegetables. 2017. 